\title{
Analysis and Improvement of Car Interior Roaring Noise
}

\author{
Shuhua Liao ${ }^{1, a, *}$, Xu Zhang ${ }^{1, b}$, Li Liu ${ }^{2, c}$, Guobin Liu ${ }^{3}$, Zhujian Chen ${ }^{3}$ \\ ${ }^{1}$ Guangxi University of Science and Technology, Liuzhou 545000 \\ ${ }^{2}$ Guangdong Ocean University, Zhanjiang 524000 \\ ${ }^{3}$ China Automotive Technology and Research Center, Tianjin 300000 \\ àliaohsh60@qq.com, ${ }^{\mathrm{b}}$ xu461139788@163.com, 다ulicatarc@163.com
}

Keywords: 3G WOT, ODS, mode, FEM.

\begin{abstract}
There is $2500 \mathrm{rpm}$ roar in the 3G WOT acceleration, and the subjective feeling is poor. Through further investigation and verification, it is found that when the car accelerates to about 2500rpm, the ODS of the front sub-frame is basically consistent with the first order modal shape of $83.6 \mathrm{~Hz}$, which was the main factor causing the roar of the car. In order to solve this problem, the idea of the equivalent method of the car body boundary is introduced to carry out FEM and the improved program is put forward. Finally, the improved program effectively solved the problem of acceleration roar at 2500rpm.
\end{abstract}

\section{Introduction}

With the improvement of living standards, the customer's demand for automotive NVH performance is also higher. Front sub-frame as an important part of the car chassis system, axles and suspension are connected to the body through it. And as the connection between the powertrain and body, it is also the important support parts of the engine. When the car driving, the front sub-frame will bear the load including road and engine vibration load and so on, when the exciting frequency is close to the natural frequency of the front sub-frame, the resonance phenomenon occurs, vibration transmitted to the entire body, it will affect the ride comfort of the vehicle ${ }^{[1]}$.

For the NVH performance of the car, 3G WOT(3rd gear wide open throttle) acceleration is a frequently investigated condition. When developing a new car and evaluating the noise inside the car, we can quickly test the noise level of the car in $3 \mathrm{G} \mathrm{WOT}^{[2]}$. Existing prototype car NVH development, there is a clear roar near the 2500rpm, and subjective feeling is poor. Taking the prototype car as an example, this paper research the problem of accelerating roar, combined with test and simulation, and ultimately eliminate the roar.

\section{Analysis of Problems}

Test conditions: in the road test, test site selection should be a relatively quiet environment (environmental noise lower than the measured noise more than $10 \mathrm{~dB}$ ), the ground is relatively flat asphalt road, collecting data in 3G WOT. The engine crankshaft speed tracking range is 1000rpm to 3500rpm. Test load for the half-load state, that is, the Trimmed Body, a data collection engineers, a professional driver. In order to ensure the reliability of the data, it is necessary to collect more than three times with the same condition, and average data with better consistency.

Test results shown in Figure 1, the driver's right ear OASPL(overall sound pressure level) curve peak is near 2500rpm in the 3G WOT, where the peak corresponds to the subjective evaluation of the roar. The order of the driver's right ear OASPL is analyzed and it is found that the second order noise occupies the main contribution. Analysis of the driver's right ear Waterfall diagram is shown in 
Figure 2, the results are consistent with the above analysis, that is, the second order noise plays a leading role in 2500rpm OASPL.

According to the relationship between engine speed $n$, order $i$ and noise frequency $f$ :

$$
\mathrm{f}=\mathrm{n} / 60 \times \mathrm{i}
$$

According to formula (1) $\mathrm{f}=83.3 \mathrm{~Hz}$, it is necessary to focus on the body structure of about $83 \mathrm{~Hz}$ resonant frequency.

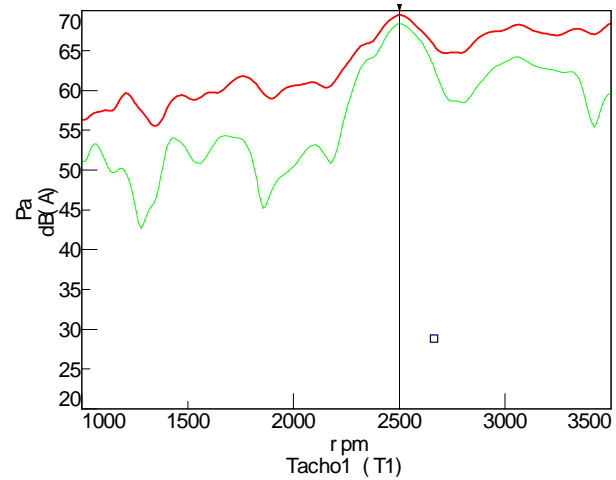

Fig.1 3G WOT driver's right ear OASPL and second order noise

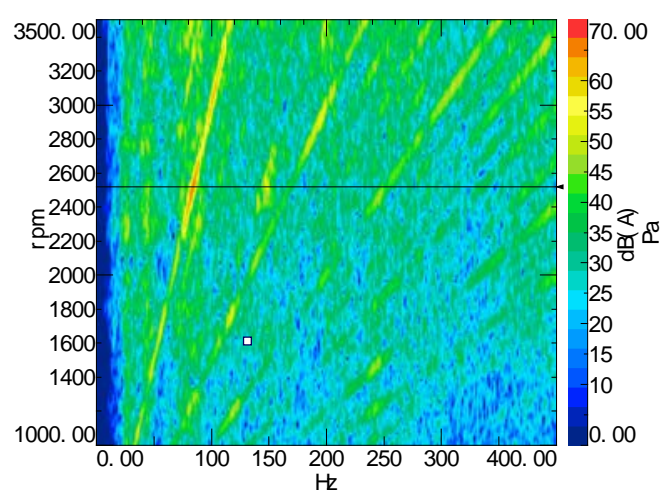

Fig.2 3G WOT driver's right ear Waterfall

\section{Problem Point Confirmation}

To further determine the problem point, the vibration of the engine mount active end and the passive end is first investigated. The investigation found that the rear mount passive end exists 2500rpm vibration peak, as shown in Figure 3. Because the vehicle engine rear suspension and body connected by the front sub-frame, it is suspected that the roar in the car is caused by the vibration of the front sub-frame.

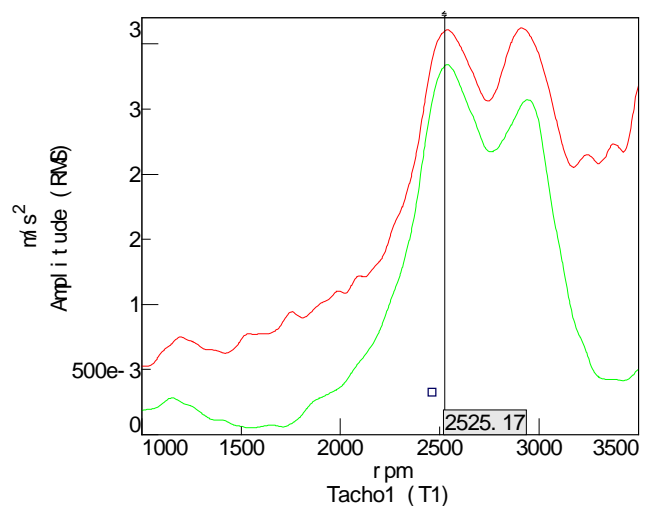

Fig.3 3G WOT rear mount passive end vibration

\subsection{Front Sub-frame Modal Test}

In order to confirm the existence of $83 \mathrm{~Hz}$ mode of the front sub-frame, modal test is carried out on the vehicle front sub-frame. In the installation state, the modal test results of the front sub-frame are obtained by testing. The test is carried out in a single-input and multi-output mode. Since the front sub-frame has a rear mount installation point, it is an input point during the hammering. Sensor arrangement and test results shown in Figure 4, the results show that front sub-frame first order mode frequency is $83.6 \mathrm{~Hz}$.

\subsection{Sub-frame ODS Test}

ODS (operational deflection shapes), refers to the actual work deformation of the tested component at a specific frequency, specific speed or a specific time, it describes the forced vibration deformation of the tested component under actual work excitation ${ }^{[3]}$. In practical applications, the modal shapes and the ODS are compared to find out which modes are the main factors causing the deformation of the work, thus providing the basis for analyzing and solving the problems. 
The ODS vibration response test point of the front sub-frame is consistent with the modal test point. The engine crankshaft speed tracking range is 1000rpm to 3500rpm in 3G WOT. The ODS test result of the front sub-frame is shown in Figure 5. According to the modal test of the front sub-frame and the analysis result of ODS test, when the car accelerates to 2500rpm, sub-frame ODS and $83.6 \mathrm{~Hz}$ first order mode is consistent basically. It is obvious that the $83.6 \mathrm{~Hz}$ mode of the front sub-frame contributes greatly to the 2500rpm roar noise. Therefore, the front sub-frame could be optimized and improved through CAE analysis.
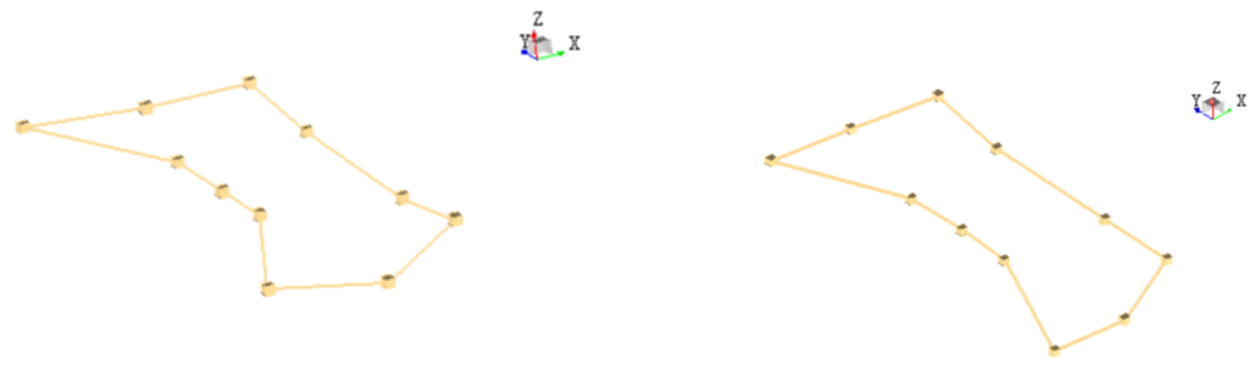

Mode $1: 83.5610 \mathrm{~Hz}, 6.00 \times$

Fig 4 Front sub-frame first order modal test results $83.6 \mathrm{~Hz}$

\section{Modal Analysis of CAE of Sub-frame}

The 3D solid model of the front sub-frame is imported into HyperMesh, and the model is meshed with shell element and solid element. In the front sub-frame model, some key points, such as mounting point of the engine mount, sub-frame mounting point and swing arm mounting point, are connected to the nodes around the hole through the RBE2 unit. The front sub-frame finite element model is shown in figure 6.

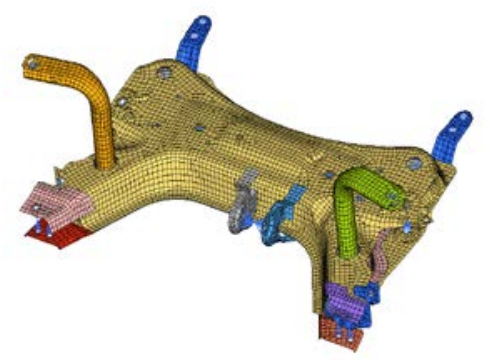

Fig 6 Front sub-frame finite element model

The front sub-frame is rigidly connected to the vehicle body by bolts, but because of the low stiffness of the body connection position, it is not advisable to consider the vehicle body boundary as a rigid constraint. Based on the equivalent method of the car body boundary ${ }^{[4]}$, the boundary condition of the connection position between the vehicle body and the front sub-frame can be simulated by introducing stiffness value of the connection point into the BUSH unit, as shown in Figure 7. The results obtained by this method show that the first order modal frequency is $84.2 \mathrm{~Hz}$, as shown in Figure 8, the error with the test mode is small and within the acceptable range. 


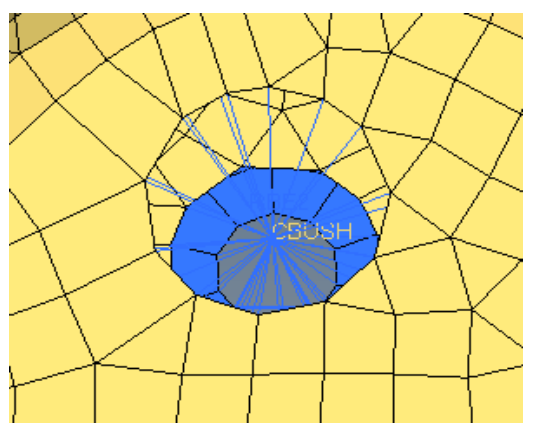

Fig 7 The simulation boundary condition of BUSH element

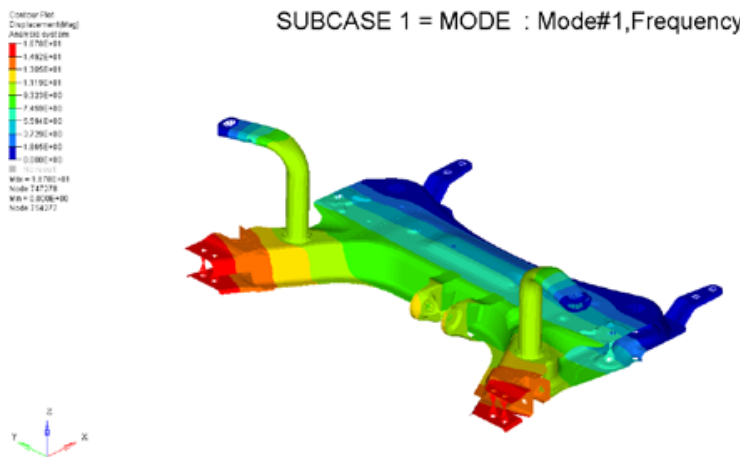

Fig 8 The first order modes in BUSH constrained state $84.2 \mathrm{~Hz}$

\section{Improvement Program}

In order to save costs and facilitate the implementation of the program to verify, the program is only based on the original structure to improve.

Program 1: Add two rectangular tubular supports to the top of the front sub-frame, as shown in Figure 9 Program 1, the thickness is $2 \mathrm{~mm}$. The first order mode is increased to $152.3 \mathrm{~Hz}$.

Program 2: Add two rectangular tubular supports to the front end of the sub-frame, so that the front sub-frame is connected with the longitudinal beam of the body through the support, as shown in Figure 9 Program 2, the thickness is 3mm. The first order mode is increased to $116.4 \mathrm{~Hz}$.

Program 3: Combined with Program 1 and Program 2, as shown in Figure 9 Program 3. The first order mode is increased to $156.8 \mathrm{~Hz}$.

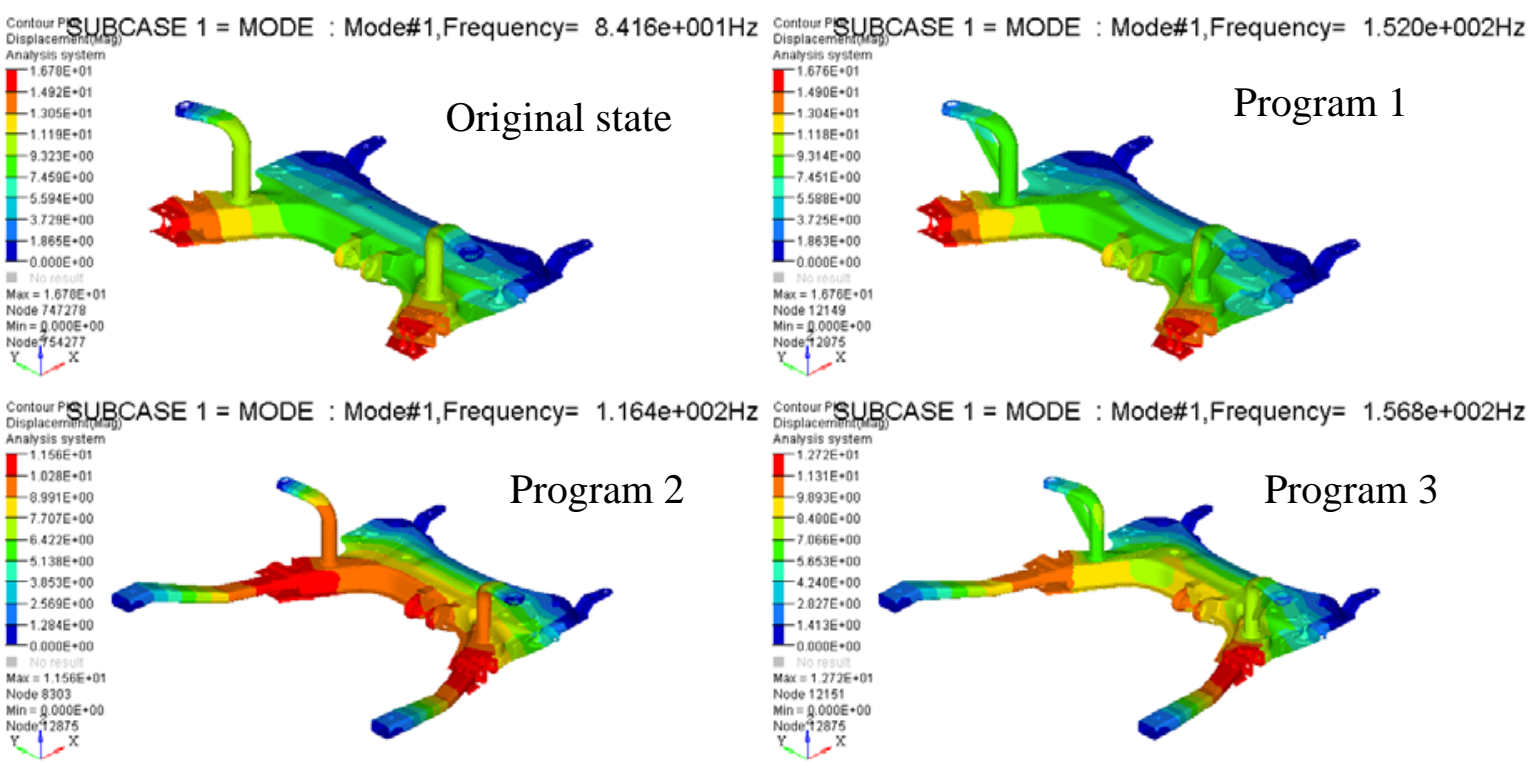

Fig 9 Improved program comparison 
Considering the above mentioned Program 1 and 2 and 3, Program 3 modal frequency increase is larger than Program 1, but the modal frequencies have little difference. Taking into account, the Program 1 is more simple and cost saving, so the Program 1 is adopted.

\section{Sub-frame Improvement Program Validation}

Restructuring front sub-frame according to Program 1, and installing the restructured sub-frame on the original car, as shown in Figure 10. The validation results are shown in figure 11. The noise peak at 2500rpm is reduced by $4 \mathrm{~dB}$, subjective feelings are better than before, the roar disappears.

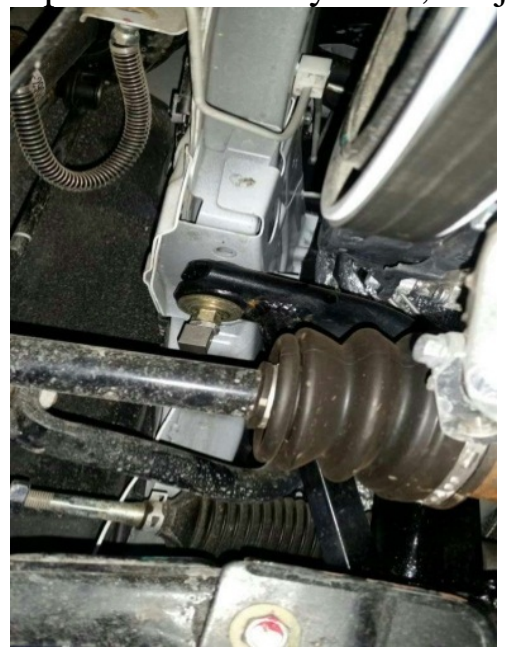

Fig 10 Restructured sub-frame

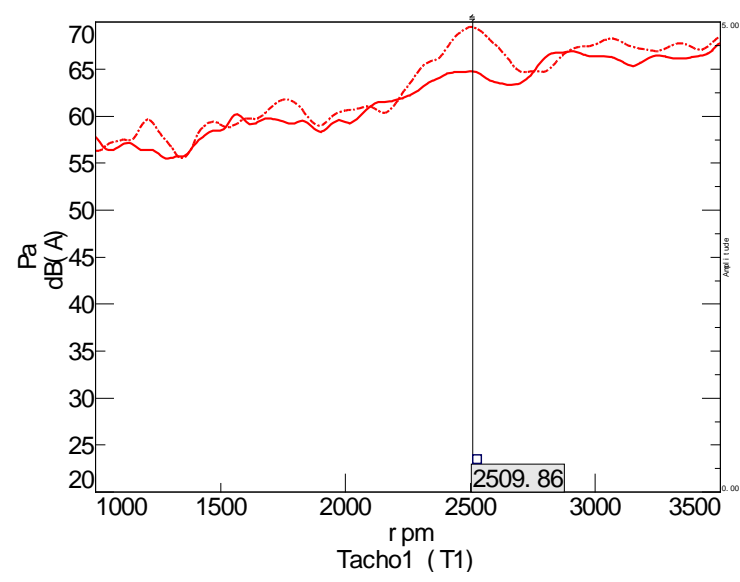

Fig 11 Comparison of validation results

\section{Summary}

In this paper, the modal test analysis module and the ODS analysis module are used to find out the structural noise source accurately for the roaring problem in the acceleration process of the vehicle. Then, CAE is used to improve the structure of the front sub-frame. After adopting the improvement measures, the noise is greatly reduced and the ride comfort is improved.

\section{References}

[1] Song Haisheng, Shi Wenku, Guo Fuxiang, Shen Zhihong, Fang Deguang. Based sub-frame car floor vibration control analysis and optimization [J]. Automotive Engineering, 2010,07: 601-604 + 610.

[2] Cheng Zhiwei, Chen Hanshuang, Zhou Guixin. Improvement of interior noise during acceleration with dynamic vibration absorber mounted to front sub-frame [J]. Auto Sci-Tech, 2014,(04): 50-54.

[3] Zhang Aidong, Chang hui, Deng Xiaolong. Application of Test.Lab modal and ODS test analysis in a vehicle roar problem [C]. 2012 LMS China User Conference Proceedings. 2012: 1-4.

[4] Gao Jidong, Chen Daliang, Li Hongliang, Wang Haiyang. Investigation on equivalent method of front sub-frame boundary conditions of passenger car for computational modal analysis [J]. Auto Sci-Tech, 2016,(02):42-44+54. 